\title{
Visual Customization Reporting System for Power Grid Dispatching based on Parsing Template
}

\author{
Zhouyang HU1, a, Xiaochen LAl ${ }^{1, b}$, Jianjian SHEN ${ }^{2, c}$, He JIANG ${ }^{1, d}$ \\ ${ }^{1}$ School of Software, Dalian University of Technology, Dalian Liaoning 116620, China \\ ${ }^{2}$ Institute of Hydropower System and Hydroinformatics, Dalian University of Technology, Dalian \\ Liaoning 116024, China
}

ahuzhouyang_leo@163.com, blaixiaochen@dlut.edu.cn, cshenjj@dlut.edu.cn, djianghe@dlut.edu.cn

Keywords: dispatching management system;parsing template;visual customization for report;data acquisition interface; component technology

Abstract. Aiming at the problem that the power network dispatching management system has the problems such as poor report expansibility, diverse data sources and complex functions, a visual customization scheme for power generation dispatching report is proposed. Based on the parsing template, using data interface technology to solve the problem of multiple data sources, component technology for report function to solve the problems of complex report, completed the design of power grid dispatching visual customization reporting system, implementation of the report template customization, report page display and other functions. The system is successfully operating in Yunnan power grid has effectively improved the power network dispatching analysis and work efficiency.

\section{Introduction}

The power network dispatching management system ${ }^{[1]}$ undertakes the provincial power grid information reporting, information dissemination and query plan, audit approval, power station unit overhaul plan to declare work, but also undertakes responsibility of providing users all kinds of statistical reports. With the development of electric power business, system needs to deal with report increase in the number of quantity and kind, which makes the system may have the following problems when add a new report demand, poor report expansibility, adding new report workload is big, need to write client and server code; diverse data sources, report data may be obtained from multiple data sources; complex functions, containing many functions such as data display, data reporting, approval etc.; can't work at any time, unable to add the required reports on the system at any time, need to wait for report code implementation; professional, need professional personnel to develop. A new report demand which is difficult to handle in time, big workload, time-consuming affects normal expansibility efficiency of the power grid business.

As reporting system is increasingly popular, at home and abroad there are two main ways to realize report system. First, using a traditional programming language to implement report system, such as the Web report system based on Spring $\mathrm{MVC}^{[2]}$, using hand coding to implement the client-side code and the server-side code. But this method needs a lot of programming and processing of data. Two, using Web reporting tools, system based on component ${ }^{[3]}$, using designer to design report structure, choosing data set in the database to be the report data sources. This method separates the custom report and report generation and it improves the usability of the system, integration, and extensibility of the system. However, using this method, the report data of the system are all derived from the local database, and can not display the third party data source information. The system has only view functions, there is no data reporting, approval etc.. With the development of science and technology, the development of Report Designer has become more mature, such as Crystal Report, iReport $^{[4]}$, jasperReport ${ }^{[5]}$, FineReport and Smartbi. The emergence of Report Designer, greatly enhance the web reporting efficiency, Excel interface style, multiple source and form backfill function conform to the Chinese report. But using Report Designer takes the purchase cost and the cost of learning. Rely too much on Report Designer and Report Engine, unable to add report anytime and anywhere. 
In view of the existing system has problems while using ready-made report design tool can not satisfy functional requirements needed for the grid business. In this paper we propose a scheme called visual customization reporting system for power grid dispatching based on the parsing template(hereinafter referred to as visual customization reporting system) to provide users with customized visualization page.We summarize our contributions as follows:

1.We propose a scheme that through report page abstract to extract statements in common, the diversity regard as the parameter with simple configuration on customized visualization page. With parameters, the abstract of report instantiate a specific report. The reporting scheme provides a new way for developers to develop the report system.

2.According to the scheme, we implement visual customization reporting system based on the parsing Excel template ${ }^{[6]}$. About 63 percent of reports are quick custom implementation. With the system the user customizes report, no need developers to write additional code, the new report added without having to restart the server, effectively improve the efficiency of the system to add new reporting requirements, reduce the developers' work time.

3.The system we design and implement of has been running in the power grid company.

The paper is organized six parts. First part, introduce the system background and the development of report system background. Second part, we present the results of report style statistics in the existing system, the generality of report and scheme. Third part, present the design of system structure and describe the design of two modules of system. The fourth part, describe the implementation of visual customization reporting system. The fifth part, discuss actual operation of the system, and finally, The last part, conclude and describe some future work.

\section{Statistics and Scheme Design}

The main idea of this paper is to solve the problem of poor report expansibility, the variety of data sources and the complex function of report forms when need to add new report demand. In order to make the scheme effectively solve the problems, before scheme designed, we find the common characteristics of the report from structure of report, report function, report data source etc. by statistic and analysis of the report in the existing system.

Table 1 shows the result of report style statistics in existing systems. From the table, we can find that most of the report style is the row expansion while column expansion style is only 4, and the other styles are rare. While the implementation of the column expansion style report can be regarded as the transposition of row expansion style report.

Table 1: Existing system report style statistics

\begin{tabular}{ccc}
\hline report style & style characteristics & number \\
\hline row expansion & first col as power plants; the first few rows as data name & 46 \\
column expansion & first row as power plants; the first few cols as data name & 4 \\
cell expansion & data and data name appear alternately & 1 \\
complex style & nested table;. multi-table synthesis & 1 \\
\hline
\end{tabular}

Row expansion style report structure is basically the same, the report page can be divided into four parts: page header information, report name, query function buttons and data tables, as shown in Figure 1. The data table is a two-dimensional table, consisting of header and data. Start with the report header fixed, one row for a power plant related data, the number of rows increases with an increase in the number of power plants.
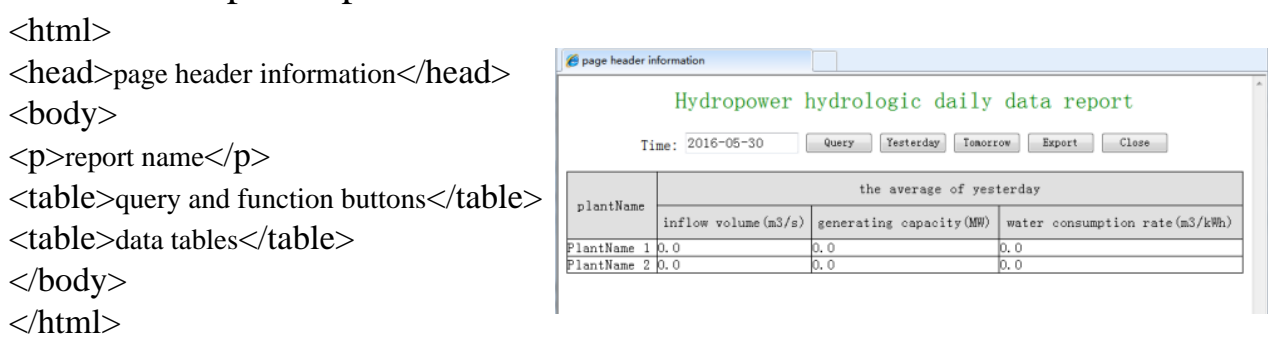

Figure 1: Structure of General Report 
How to fast realize the four parts of the report page is the key to improve the efficiency of the system. The four parts of the report page have the common character, to add new report as an abstract problem, then the system demand for adding new report is converted to identify four parts.

In addition to the general functions of the report system, there are some functions such as "report contents summarization ${ }^{[7] ", ~ " e x t r a c t ~ t h e ~ p l a n n e d ~ v a l u e ", ~ " g e n e r a t e ~ g r a p h ", ~ " c o l o r ~ t o ~ t h e ~ i l l e g a l ~ d a t a " ~}$ and so on. Table 2 lists the top 5 general function of the report in the system. To implement these functions can complete the function of the vast majority of reports.

Table 2: statistics table of existing system report function

\begin{tabular}{cc}
\hline rank & report function \\
\hline 1 & data query \\
2 & export data, download \\
3 & save data(data report or data modification) \\
4 & data verification \\
5 & download report template, browse to upload \\
\hline
\end{tabular}

In the existing system, the report data mainly comes from three forms: data reporting, data accessed by JDBC and the third party data interface. How to realize data acquisition of multi-source data report is the key to improve system performance.

With the analysis of the report style, the report function and the data source of the existing system, the design of the specific visual customization reporting program as follows:

1. Conduct the data reduction in the report page ${ }^{[8]}$, extract report page in common, get the needed for configuration.

2. Design the report structure quickly by component or report design tool, and reduce the coding of the new report structure.

3. Achieve the functional components of reports, reports required functions call function directly.

4. Set up the data source for the report header columns, data directly obtained by the data source. Don't need to code the data acquisition function for every report and reduce code redundancy.

5. Visual customization page, configure the information contained in the above four steps.

\section{Visual Customization Reporting System Design}

Visual customization reporting system is based on $\mathrm{J}_{2} \mathrm{EE}^{[9]}$ structure, using B/S framework ${ }^{[10]}$. The overall structure of the visual customization reporting system is divided into four layers: presentation layer, control layer, business logic layer and database service layer, as shown in Figure 2. The presentation layer uses some lightweight frameworks technology (jQuery, Ajax, etc.) to realize the

\begin{tabular}{|c|c|c|c|c|c|c|}
\hline \multicolumn{7}{|c|}{ Browser(IE,FireFox,Google Chrome) } \\
\hline presentation layer & $\mathrm{jQ}$ & uery & Ajax & HTML & CSS & \\
\hline control layer & & Servle & & Jsp & Cache ) & \\
\hline business logic layer & \begin{tabular}{|l|} 
obtain \\
report \\
structure \\
\end{tabular} & $\begin{array}{l}\text { obta } \\
\text { infoo }\end{array}$ & $\begin{array}{l}\text { basic } \\
\text { nation }\end{array}$ & $\begin{array}{l}\text { local } \\
\text { data } \\
\text { access } \\
\end{array}$ & \begin{tabular}{|l|}
$\begin{array}{l}\text { examination } \\
\text { and approval } \\
\text { information } \\
\text { access }\end{array}$ \\
\end{tabular} & \begin{tabular}{|c|} 
obtain \\
other data \\
source \\
\end{tabular} \\
\hline $\begin{array}{ll}\text { database server } \\
\text { layer }\end{array}$ & $\begin{array}{l}\text { report } \\
\text { citure and } \\
\text { h col data } \\
\text { source }\end{array}$ & $\begin{array}{l}\text { basi } \\
\text { inform }\end{array}$ & & local data & $\begin{array}{l}\text { examination } \\
\text { and approval } \\
\text { information }\end{array}$ & $\begin{array}{l}\text { other data } \\
\text { (The third } \\
\text { party data) }\end{array}$ \\
\hline
\end{tabular}

Figure 2: system structure

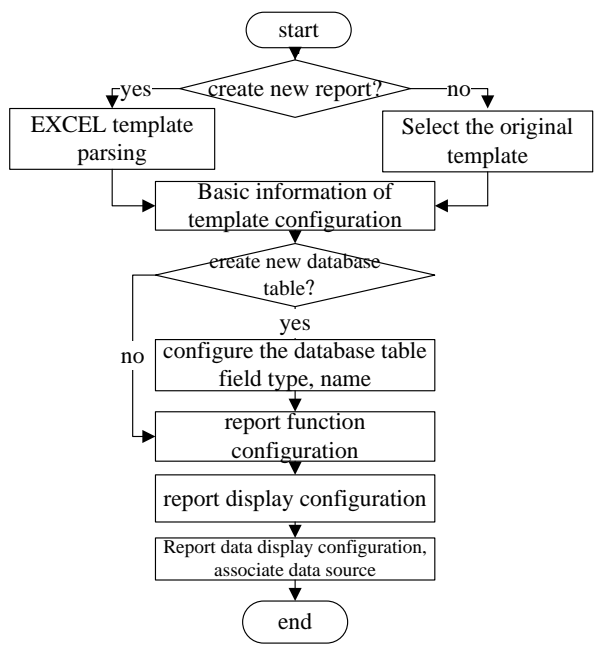

Figure 3: report customization flowchart functions of the foreground generic buttons, reduce code duplication of button functions and the workload of new reports, increase the scalability of the system. Control layer is responsible for receiving all kinds of request information, calling the business module and returning the results to 
each request source. It controls the process of responding, retrieving, and displaying reports. The business logic layer receives the request of the contents according to the control layer, and get the report structure and the information of the report and the result is packaged as an object back to the control layer. Database service layer uses Oracle $10 \mathrm{~g}$ as the primary data platform to provide various types of data services. Use $\mathrm{H} \mathrm{Base}^{[11]}$ to store all kinds of fast-growing actual operating data like real-time water level value and real-time output value. Use the third party data interface to get data of other system. With the JDBC database connection pool ${ }^{[12]}$ and data acquisition interface to achieve the data saving and taking.

According to the system function, visual customization reporting system is divided two modules: 1.report customization and management module; 2.report display pages nodule.

Report Customization and Management Module Design. Report customization and management module is composed of two modules: report customization module and management module.

Report customization module provides users with personalized information configuration services. According to the former chapter, one report page includes page header information, report name, query function buttons and data tables. In this module, it provides configuration interface for those four parts. The module flowchart is shown in Figure 3.

In this paper, we use Excel to provide report structure template for the system. The system parses the Excel file and saves the report structure information to be used by report display. In the Excel, there is just the report structure and no data. Configure page header information and report name in the "basic information of template configuration", when in "report function configuration", we will provide function list to choose from. If add a fixed column for the report or merge cells for the first $\mathrm{N}$ columns, choose the option in "report display configuration". The last step, configure data source for each valid column which the header cell near the data area and assign the display mode to the column. If the report needs data reporting, users can directly create the database table on the page, rather than using the database management system.

In management module, users can delete the database table which created for holding data and delete the report which created in report customization module.

Report Display Pages Module Design. We design two main web pages for report display. One displays the links of all reports which have been completed in report customization and management module. The name of the link is report name and the URLs are similar, while the only difference is that their parameters which are the the identity of the report are different. Another displays the report. This page is divided into four parts: load page header information and report name, load query button and other function buttons, load report and get the data form.

\section{Implementation of Visual Customization Reporting System}

Implementation of Report Customization and Management Module. Due to the number of the column expansion style report is small from Table 1, and the implementation of the column expansion style report can be regarded as the transposition of row expansion style report, this paper implements the customization of the row expansion report.

(1) Parsing Excel Template

Use Excel to make the report template, and parse template to get report structure. The realization of the parsing template process as shown in Figure 4. Each cell has a number listed in the Excel that "ABC... Z AA AB..." is the column number, while the number "123..." is the row number. Put the column number and row number together to synthesize a coordinate that is the position of the cell, a sample is shown in Figure 4 (a). Use JXL library a java tool to operate Excel, the coordinates of the upper left corner and the lower right corner of the cell are recorded, the two coordinates "position1@position2" indicates the position of a cell in Excel. Position1 is the coordinate of the upper left corner of the cell while position2 means the coordinate of the lower right corner of the cell, as shown data1 in Figure 4 (b). Data 2 is the name list of each cell. The results of the parsing excel templates are saved to the database, as shown in Figure 4 (c) and (d). Each Excel template has its own 
id a unique code to distinguish between different reports. The implementation of the parsing template process as shown in Figure 4.

\begin{tabular}{|c|c|c|c|c|c|c|c|c|}
\hline & A & B & $\mathrm{C}$ & D & \multirow{2}{*}{\multicolumn{4}{|c|}{$\begin{array}{c}\text { data1 : A1@A2;B1@D1;B2@B2;C2@C2;D2@D2 } \\
\text { data2: column name1;column name2;column name3;column name4;column name5 }\end{array}$}} \\
\hline \multirow[b]{2}{*}{2} & \multirow{2}{*}{$\begin{array}{l}\text { column } \\
\text { name1 }\end{array}$} & \multicolumn{3}{|c|}{$\begin{array}{l}\text { column } \\
\text { name2 }\end{array}$} & & & & \\
\hline & & $\begin{array}{c}\text { column } \\
\text { name3 }\end{array}$ & $\begin{array}{l}\text { column } \\
\text { name4 }\end{array}$ & $\begin{array}{c}\text { column } \\
\text { name5 }\end{array}$ & \multicolumn{4}{|l|}{ template parsing } \\
\hline \multicolumn{5}{|c|}{ (a) reportl's excel template } & \multicolumn{4}{|c|}{ (b) results } \\
\hline & UMENT_ID & ARGUMENT_NAME & ...... & REPORT_ID & & & 1] $\begin{array}{c}\text { save to the } \\
\text { database }\end{array}$ & \\
\hline & 1 & column namel & & 1 & REPORT_ID & REPORT_NAME & ARGUMENT_LIST & ....... \\
\hline & 3 & column name3 & …. & 1 & 1 & Report name 1 & $\mathrm{~A} 1 @ \mathrm{~A} 2 ; \mathrm{B} 1 @ \mathrm{D} 1 ; \mathrm{B} 2 @ \mathrm{~B} 2 ; \mathrm{C} 2 @ \mathrm{C} 2 ; \mathrm{D} 2 @ \mathrm{D} 2$ & $\ldots \ldots$ \\
\hline & $\begin{array}{l}4 \\
5\end{array}$ & column name 4 & & 1 & & & & \\
\hline & & column name5 & 然 & 1 & & & result of datal in the database & \\
\hline
\end{tabular}

Figure 4: template parsing process flow diagram

(2) The Configuration of The Information of Report Template

According to the configuration content, the report customization page is divided into three areas. From top to bottom of the page, the three areas are information configuration area, report structure displaying area and the area of configuring data source. Basic information of template, report function and report display configuration are all in information configuration area.

Basic information of template configuration includes the report name and report type. We provide the text label for the user setting the report name while provide the select label for choosing report type. The report type reflects the particularity of the industry. According to the different business of each report, the system has set up several main menus and each main menu is divided into some sub-menus and each sub-menu corresponds to a report type. The report types for choice are stored in the database, and all report types are loaded into the select label for user selection when the page is initialized. The text label for report name automatically populate the excel file name as the report name and users can change the name in the text label.

We provide three select labels for users to choose the report function. First select label is selecting query time;second select label is selecting the approval method;third select label is selecting report function. The option in labels can't be added or removed due to each option corresponds to a function method which has been prepared in advance. One page table for choosing reporting deadlines, we list some time node. If the daily report, user can choose 24 nodes as the deadlines each node represents a particular time. We provide two select labels for report display configuration. Choosing the first $\mathrm{n}$ columns to merge cells and choosing the first n columns fixed.

All the information configured in information configuration area will be sent to the server by form label and stored in the database the same database table shown in Figure 4 (c).

(3) Create New Database Table For Reporting

Report customization page has the toggle button of create new database table for reporting. When click the button, system parses the effective column name to get the column name initials as database table field name. If the column name starts with a number, add "a" before the name to generate the field name. If the column name is Chinese, we need to process the Chinese string as follows:

1.Get Chinese strings using GB2312 encoding.

2.Get the section-position code of each Chinese character.

3.Compare the section-position code of Chinese characters with the section-position code of the first Chinese character representing one letter.

So does database table name by report name. According to whether the column name contains the word with special meanings like time, value and some data units, system gives the default field type. Users also can change the filed name and type as needed. Each database table will be added a field to save the date of data. We use Ajax to submit the information of new database table and in the server side use JDBC to execute the SQL statement returning the result to the report customization page. If the database table was created successfully, the effective column will be automatically associated with the corresponding field.

(4) Report Data Display Configuration and Associate Data Source

Report data display configuration and associate data source is shown in Figure 5. 


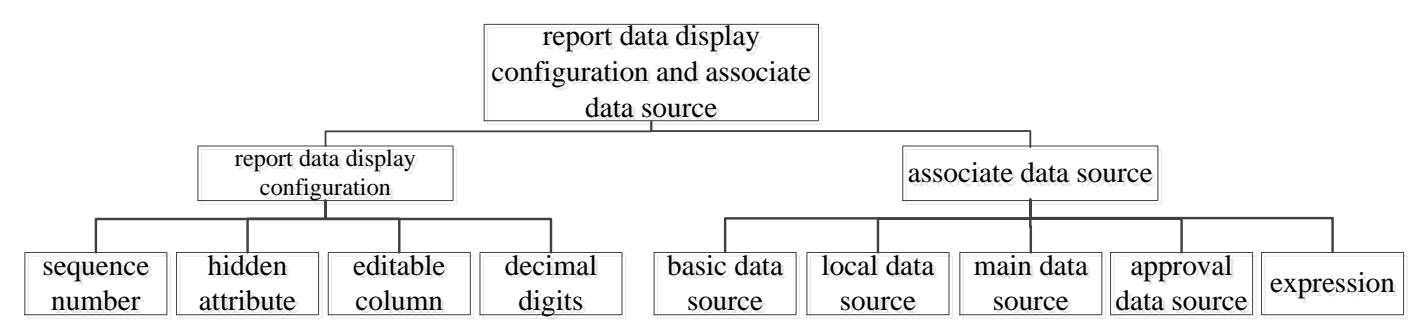

Figure 5: column data display configuration and associate data source structure

We insert an iframe to show the report header in the report structure displaying area. Each header cell can be clicked, if the cell chosen, sub-page will pass the selected cell tag value to the parent page. When the user completes the configuration of data source for the cell on the parent page, the system will post the tag value and configuration content together using Ajax to the server to save asynchronously.

The area of configuring data source is divided into three parts, choosing column data display, choosing the type of data source and choosing the parameters of the data source. If user clicks the one of the radio button of type of data source, the third part of the area automatically displays the corresponding configuration interface.

According to the display of the data of each column we provide "hidden column"(hide the column content, set "display=none" for the the style attributes of the label ), "set column value to the serial number", "set whether the column can be edited", "set the decimal digits".

Different data sources need to be configured different parameters. Basic data source, what needs to be configured are: what is the data object, what is the content of the display, which is dependent on. Local data source, choose specific database name, database table name and field name for the column. Main data source (Data that has been calculated or retrieved from a third party for reporting data), the page provides the user with the following parameters: data name, data type, data unit, statistical type, the time of data and so on. Approval data source, user can choose the approver, approval time, notes and other information. Expression, system will provide the number of each column, and user uses the number to design the expression for the configuring column, the result of the expression is the column value. Data source configuration for valid cells only.

Implementation of The Report Display Page. (1) Report Linked List

When report display module is initialized, each main menu load their own sub-menu which added as report type in management page. Each sub-menu lists reports configured the report type in the database. The pseudo-code of loading sub-menu is shown in Pseudo-code 1.

\begin{tabular}{l} 
Pseudo-code 1 main menu loads sub-menu \\
\hline Input:
\end{tabular}

L:a list report types in database, each object including type name and the number of the type

M:Menu object in page

sub-menu List: the list of sub-menu,each sub-menu including its own URL.

\section{Output:}

sub-menu List

\section{Body:}

1: $\mathrm{L}=$ loadReportTypeList()

2: for each report type $t \in L$

3: if t.number $\in \mathrm{M}$

4: add $t$ link to the sub-menu List

5: end if

6: end for

7: return sub-menu List

Each report type has type name and the number of type. If the number is within main menu's scope, the main menu loads the sub-menu. Use of JavaScript code to achieve sub menu loading method.

According to the number of sub-menu, report list area loads report links. The pseudo-code is shown in Pseudo-code 2. 
Pseudo-code 2 load report links

\section{Input:}

L:a list of report templates, each template has report id, report name and other information of report $\mathrm{N}$ : the number of sub-menu.

\section{Output:}

\section{Link-List}

\section{Body:}

1: $\mathrm{L}=$ loadReportTemplatelList()

2: for each report template $r \in \mathrm{L}$

3: if $\mathrm{r} \cdot$ reportType $=\mathrm{N}$

4: add $r$ link to the Link-List

5: end if

6: end for

7: return Link-List

(2) Component Technology for Report Function

In the report display, the report function appears in the form of a button on the report page. The report function will be implemented in advance, stored in each JS file. According to the information of configuration when the report is customized, the page loads the button that binds the function.

(3) Parsing Excel Template for Display

According to the information of the report header structure saved in database, the report display page show the report table. Take the report in Figure 4 as an example, the process of generating the report header in the report display is as follows:

1.According to the report_id the parameter of the report link, get the report header structure information recorded as "argument_list" and the name list of the cell recorded as "name_list".

2.Split "argument_list" to an array of strings separated by ";" recorded as data1,each string of data1 is the position of cell in form of "position1@position2".

3.According to the array index from small to large to traverse the array of data1, the result of the letter of the position 1 minus the letter of position 2 plus 1 is the value of cell's rowspan. The result of the number plus 1 is the value of the cell's colspan. At the same time, the position in the alphabet of the letter in position 1 is recorded as cell's row.

4.Generate HTML table statement based on the value of rowspan and colspan, when the row changes, the cell is on the new line.

(4) Data Acquisition Interface

All the data of the report is called by the data acquisition interface. Data acquisition interface implementation is shown in pseudo-code 3.

Line 2, according to the intersection of user's jurisdiction and configuration on the basic data source to determine query power plant set. At the same, according to the $\mathrm{M}$, we get the effective number of columns. If the effective number of columns is $\mathrm{n}$ and the power plant set size is $\mathrm{m}$, pre-generate storage space $m^{*} n$ for the data. The server will initialize all power plants and grid information when the system is started. The server provides an object called "powerSystem". It has getting the basic information function of each power plant and grid. According to the configuration of basic data source, system call the function in "powerSystem" to get the value of the whole column.

Line 3, according to the database name, database table name and field name generate SQL query. If some columns' database name and database table name are same, we put the fields merge into a query to reduce the number of SQL queries. All data is reported by users using system, if there is no query result, according to the type of field to initialize a value to fill in the data. Line 4 and Line 5 according to parameters, call corresponding function to get the column data. Line 6 calls getting expression data function, The existing numerical columns of simple arithmetic, the result is the value of the column.

\section{Pseudo-code 3 get selected report data lines}


T:query time

M:selected report model; object includes all configuration information

U:user;object includes user information

\section{Output:}

Data:the data of report

\section{Body:}

1: Data $=$ new Vector $<$ Vector $>()$

2: Data $=$ getBasicSourceData $($ Data $, T, M, U)$ : get the power plant set and the information of plants

3: Data $=$ getDatabaseData $($ Data, $\mathrm{T}, \mathrm{M}, \mathrm{U})$ : get the local data

4: Data = getApprovalData(Data, T, M, U): get approval data

5: Data = getMainData(Data, $\mathrm{T}, \mathrm{M}, \mathrm{U})$ : get main data

6: Data $=$ getCalculateData $($ Data, $T, M, U)$ : get expression data

7: return Data;

Lines 3 to 6 all function returns are "power plant number + value". Compare the power plant number to the column in the data which having been assigned in the Line 2 function. If the number is same, put the value on the corresponding position. When all the columns are not with some type of data source, the system will skip the source data acquisition function call.

Data reporting or data modification is the the data displaying inverse process. We just save the columns data which configured local data source. According to the chapter of "Create New Database Table For Reporting", generally one report data is stored in the same database table. Each columns has its own field name, to determine the data belongs to the first $\mathrm{N}$ columns, match the data and field name in the SQL statement. Each record adds extra power plant number and data time to facilitate data query report.

\section{Experimental Result \& Performance Analysis}

So far, the system based on the above system design scheme of power grid dispatching system has been developed and deployed applied in the power grid company. With the actual operation test the system can achieve more than $63 \%$ of reports, at the same time we still support the original report statements deployment. Report implementation rate is shown in Table 3.

Table3: report implementation rate

\begin{tabular}{cccc}
\hline report & achieved by custom & not achieved by custom & implementation rate (\%) \\
\hline original system report & 33 & 19 & 63.5 \\
other daily report & 96 & 34 & 73.8 \\
sum & 129 & 53 & 70.9 \\
\hline
\end{tabular}

Visual customization reporting system can not achieve by all reports, however, an average of $70.9 \%$ of the implementation rate can improve the efficiency of most reports.

Analysis of the time to achieve a report, customize a report will cost about 15 minutes on average, while coding for a report page may cost more than two hours. Use this system to save time to develop reports.

From the perspective of the user, A person who has been trained for 1 or 2 hours will be able to add a report using the visual customization reporting system, while if use the code to implement a report ,the developer need to understand the system-related development language.

The analysis on system personalization and running efficiency, the style of the report in system is basically the same. If the report needed some personalized features, we need add the new function to the system and when the subsequent report also need those personalized features, configure the function for the report in report customization and management module. As the report is loaded in a different way from the traditional, system need to get the report structure from the database and use JavaScript function to set the mode of display. It may cost extra time. 


\section{Conclusions}

In this work we analyze the existing power grid dispatching management system, find that when the system increases new report page, there are some problems including low expansibility, diverse data sources, reporting features complex, unable to work at any time and need professional developers to realize. We propose a visual customization reporting scheme and design and implementation of visual customization reporting system to resolve problems. With the system, users can customize the required report, no need to prepare the background code, no need to restart the server, saving the time to complete the additional report requirements. Since the system on line, we have achieved customized reports 33 accounting for $63.5 \%$. Customizing a report cost about 15 minutes on average, while the traditional writing a report cost more than 2 hours reduces by more than $80 \%$, improves the working efficiency of the demand for new report and the expansibility of the system.

As a future work, we will increase the reporting rate of the system, implement the customization of column expansion style report, recommend template snippets ${ }^{[13]}$ and solve the problem of running efficiency.

\section{Acknowledgements}

This work was financially supported by the Natural Science Foundation of China (51579029) and Fundamental Research Funds for the Central Universities (DUT16QY30).

\section{References}

[1] Gang Li, Xiaocheng Ying, Chuntian Cheng, et al: Automation of Electric Power Systems, Vol. 35-16 (2011), p. 79-81(In Chinese).

[2] F. Zhang, and S. J. Wang: Design and Implementation of a Logistics Report Management System Based on Spring MVC, 2015 International Conference on Electrical, Automation and Mechanical Engineering, Atlantis Press(2015).

[3] Ligang Tian: International Journal of Hybrid Information Technology, Vol. 8-3(2015), p. 375-386.

[4] Hiltemann, S., Hoogstrate, Y., Van Der Spek, P., et al: GigaScience, Vol.3-1 (2014), p. 19.

[5] Mei Du, Chuansheng Zhou, and Sheng-jia Cao: Journal of Shenyang Normal University (Natural Science Edition) (2007)(In Chinese).

[6] Wallace R G. , U.S. Patent 8,584,150. (2013).

[7] He Jiang, Jingxuan Zhang, Hongjing Ma, et al: Science China Information Sciences(2015).

[8] Jifeng Xuan, He Jiang, Yan Hu, et al: IEEE Transactions on Knowledge and Data Engineering, vol.27-1(2015), p.264-280.

[9] Bhatt, Manish: Journal of Global Research Computer Science (JGRCST)(2014).

[10]Waziri, U., Dan, J., Danjuma, S., et al: International Journal of scientific \& technology research, ISSN (2014).

[11] Dimiduk, N., Khurana, A., Ryan, M. H., et al: HBase in action, Shelter Island: Manning (2013).

[12] J. Du, L. J. He, and H. J. Ouyang: Journal of Xianyang Normal University, Vol. 29-2(2014), p. 26-29(In Chinese).

[13] He Jiang, Liming Nie, Zeyi Sun, et al: IEEE Transactions on Services Computing(2016). 